\title{
Abnormal arginine metabolism is associated with prognosis in patients of gastric cancer
}

\author{
Lin-Yang Shi ${ }^{1 \#}$, Yuan-Yuan Wang ${ }^{2 \#}$, Yu Jing ${ }^{2}$, Ming-Hao Xu ${ }^{2}$, Zhi-Tu Zhu ${ }^{3}$, Qing-Jun Wang ${ }^{2}$ \\ ${ }^{1}$ Department of Oncology, The First Affiliated Hospital of Jinzhou Medical University, Jinzhou, China; ${ }^{2}$ Department of Clinical Trial, The First \\ Affiliated Hospital of Jinzhou Medical University, Jinzhou, China; ${ }^{3}$ Department of Clinical Trial, Institute of Clinical Bioinformatics, Cancer Center \\ of Jinzhou Medical University, The First Affiliated Hospital of Jinzhou Medical University, Jinzhou, China \\ Contributions: (I) Conception and design: ZT Zhu; (II) Administrative support: QJ Wang; (III) Provision of study materials or patients: LY Shi; (IV) \\ Collection and assembly of data: LY Shi; (V) Data analysis and interpretation: YY Wang; (VI) Manuscript writing: All authors; (VII) Final approval of \\ manuscript: All authors. \\ \#These authors contributed equally to this work. \\ Correspondence to: Zhi-Tu Zhu. Department of Clinical Trial, Institute of Clinical Bioinformatics, Cancer Center of Jinzhou Medical University, \\ The First Affiliated Hospital of Jinzhou Medical University, No. 2, Section 5, Renmin Street, Guta District, Jinzhou 121000, China. Email: \\ zhuzhitu@163.com; Qing-Jun Wang. Department of Clinical Trial, The First Affiliated Hospital of Jinzhou Medical University, No. 2, Section 5, \\ Renmin Street, Guta District, Jinzhou 121000, China. Email: 63905220@qq.com.
}

Background: Metabolic disorder is a key factor in the occurrence and development of tumors. Metabolomics methods can explore a variety of prognostic markers for tumors.

Methods: The 454 patients included in this study comprised 92 cases of gastric cancer, 51 cases of gastric ulcers, 206 cases of gastric polyps, and 105 cases of gastritis. The plasma levels of 23 amino acids in patients before treatment were detected by liquid chromatography-tandem mass spectrometry, and t-test was used to determine the difference of amino acids levels between the gastric cancer group and other groups. Shared different amino acids were selected to analyze their relationship with staging, differentiation and prognosis. The TCGA database was used to explore the changes of genes expression related to the synthesis and degradation of different amino acids, and the relationship between the genes and stage, differentiation and prognosis.

Results: The plasma arginine level in the gastric cancer group was significantly higher than that in the gastric ulcer, gastric polyp, and gastritis groups ( $\mathrm{P}$ values 0.0065, 0.0306, 0.0004, respectively).The level of plasma arginine in patients with non-metastatic gastric cancer was significantly higher than that in patients with metastatic gastric cancer $(\mathrm{P}=0.0013)$. Compared with the normal control, the key metabolic enzyme ASS1 gene was highly expressed in gastric cancer, and the survival time of gastric cancer patients with high expression of ASS1 was longer. Patients with high arginine expression had significantly longer survival (logrank test $\mathrm{P}=0.0003)$.

Conclusions: Increased plasma arginine level in gastric cancer patients was related to overexpression of ASS1 by TCGA database analysis. High expression of ASS1 prolonged the overall survival of gastric cancer patients, and the arginine level before treatment could be used as a prognostic factor.

Keywords: Gastric cancer; metabolomics; arginine; prognosis

Submitted Apr 06, 2021. Accepted for publication May 21, 2021.

doi: $10.21037 /$ tcr-21-794

View this article at: http://dx.doi.org/10.21037/tcr-21-794 


\section{Introduction}

Gastric cancer is one of the most common cancers in the world, and also a common cause of death in cancer patients (1). The morbidity of gastric cancer is regional, and its prevalence in the East Asian population is higher than in European and American populations (2). There is a high incidence of gastric cancer in China, and its morbidity and mortality rank second among cancers, making it a major health issue nationally.

Metabolic disorders are a key factor in the occurrence and development of cancer, and cancer patients commonly have metabolic abnormalities (3). Therefore, the relationship between metabolomics and cancer diagnosis, prognosis analysis, and even targeted therapy has become a core topic of cancer research in recent years. From the perspective of evidence-based medicine, the development of gastric cancer is a gradually evolution from precancerous lesions to malignancy. During this process, the growth of cancer tissue demands energy, leading to rearrangement of metabolites. Several studies have reported that the plasma metabolic profiles of patients with gastric cancer are different from those of patients with benign gastric disease (4-6). Therefore, some metabolites are considered to play an important role in different aspects of gastric cancer $(7,8)$, which is helpful for exploring the relationship between the prognosis of gastric cancer and metabolism.

Arginine is a non-essential amino acid in the human body. In addition to being used for protein synthesis, it also participates in many metabolic pathways as a precursor of other substances, such as nitric oxide, polyamines, spermine, etc. (9). Arginine and these downstream substances are involved in tumor growth, proliferation, invasion, metastasis and angiogenesis. In recent years, it has been reported that arginine is a sensitive marker for the development of breast cancer, prostate cancer and other tumor species $(10,11)$. Abnormal expression of arginine in gastric cancer models has been found in cell and animal experiments (12). There have been previous reports of abnormal metabolism of arginine in patients with gastric cancer (4), but no reports of analyses of arginine and the prognosis of gastric cancer.

In the urea cycle with the participation of arginine, citrate and aspartate form argininosuccinate catalyzed by argininosuccinate synthetase (ASS1), and argininosuccinate forms arginine under the action of argininosuccinate lyase (ALS). However, ASS1 is the key rate-limiting enzyme for the production of arginine. In different types of cancer, the ASS1 gene is either overexpressed or absent. In summary, changes in the levels of ASS1 lead to disorders in arginine metabolism, leading to different prognoses.

The Cancer Genome Atlas (TCGA) database is a free and open genetic database, established by the National Cancer Institute and the National Human Genome Research Institute. Researchers can download genetic data by logging into the TCGA database website, and further analyze the data according to the purpose of the research. Although there are studies from different methods such as genomics, proteomics, etc., to analyze the differences between gastric cancer and gastric benign diseases, and to explore prognostic markers. However, there are very few studies using metabolomics to find amino acid markers for the prognosis of gastric cancer.

We present the following article in accordance with the REMARK reporting checklist (available at http://dx.doi. org/10.21037/tcr-21-794).

\section{Methods}

\section{Study population and study design}

A total of 454 patients diagnosed with gastric cancer, gastritis, gastric ulcer, and gastric polyps at the First Affiliated Hospital of Jinzhou Medical University from November 2015 to April 2020 were identified retrospectively and enrolled. All procedures performed in this study involving human participants were in accordance with the Declaration of Helsinki (as revised in 2013). This study was approved by the institutional ethics committee of The First Affiliated Hospital of Jinzhou Medical University (No. 202058). All enrolled patients provided written informed consent for their specimens to be used for research purpose. Patients with gastric cancer were confirmed by gastroscopy or postoperative pathology, and patients with benign gastric diseases were confirmed by gastroscopy examination. Blood samples were collected from all patients after fasting for at least $12 \mathrm{~h}$ before treatment to measure plasma amino acids, tumor markers including carcinoembryonic antigen (CEA), carbohydrate antigen 19-9 (CA19-9), carbohydrate antigen 72-4 (CA724) and other laboratory indicators. The clinicopathological information, including age, sex, height, weight, site of onset, TNM stage, metastatic location, differentiation and Lauren typing, were also recorded.

The exclusion criteria of this study are as follows: (I) comorbidity of other tumors or personal history of malignant tumors; (II) metabolic diseases such as diabetes 
Clinical pathological information and laboratory test results including metabolomics data were extracted from 578 patients diagnosed with gastric cancer, gastritis, gastric polyps, and gastric ulcer in the First Affiliated Hospital of Jinzhou Medical University

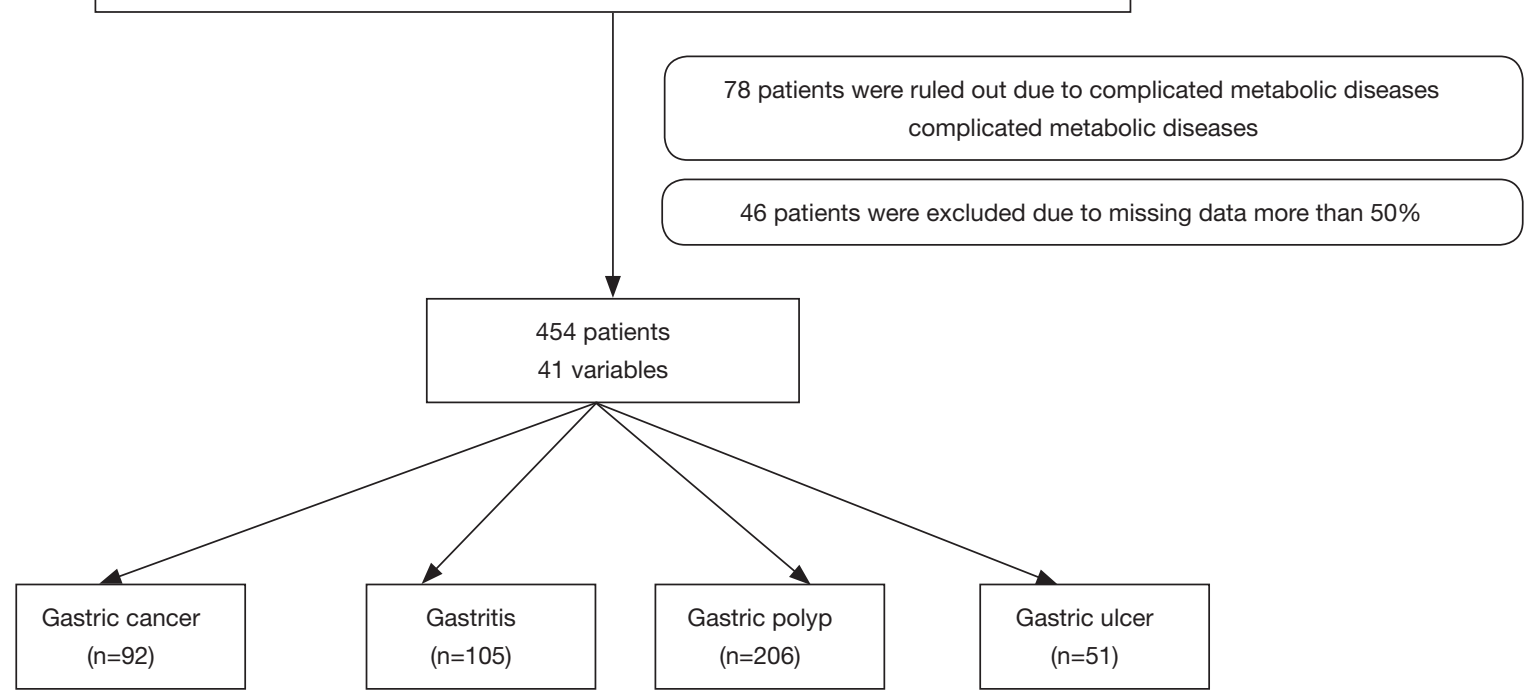

Figure 1 Patient flow diagram.

and hyperthyroidism; (III) recent persistent diarrhea or vomiting; (IV) pregnant women, children, drug or alcohol addiction. Patient collection is shown in Figure 1, and the characteristics of the study population are shown in Table 1.

\section{Chemicals}

High-purity water and acetonitrile (high-performance liquid chromatography grade) were purchased from Thermo Fisher (Waltham, MA, USA). Acetyl chloride and 1-butanol were commercially provided by Sigma-Aldrich (St. Louis, MO, USA). Internal standards of 12 isotopically labeled amino acids (NSK-A) and 8 isotopically labeled acylcarnitines (NSK-B) from the Cambridge Isotope Laboratory (Tewkesbury, MA, USA) were used to achieve absolute quantification. Mix and dissolve all the standard solutions in $2 \mathrm{~mL}$ of pure methanol and stored at $4{ }^{\circ} \mathrm{C}$. Prepare working solutions by 100 times dilution to extract the metabolites. Chromsystems (Graffenfen, Germany) provided quality control (QC) standards for amino acids and carnitine. The QC samples were regarded as authentic samples and according to provided instructions for processing to guarantee the stability of the analysis.

\section{Sample preparation}

In the morning, fasting blood samples of all enrolled patients were collected, stored in EDTA vacuum tubes, centrifuged at $3000 \mathrm{~g}$ for $10 \mathrm{~min}$ at $4{ }^{\circ} \mathrm{C}$, and then the supernatant (i.e., plasma) was transferred to a new bottle and immediately frozen $\left(-80^{\circ} \mathrm{C}\right)$ until sample analysis. Under QC and system adjustment, the QC samples were prepared by mixing all samples in an equal volume of $10 \mu \mathrm{L}$, and all tests were performed at room temperature. First, from each blood sample rushed out of the $3 \mathrm{~mm}$ (diameter) of the disc and placed in a Millipore MultiScreen HV96-well plate (Millipore Billerica, MA, USA) to extract metabolites. Next, $100 \mu \mathrm{L}$ of working solution was added to each well containing the disc, and after gently shaking slowly for 20 min, the plate was centrifuged at $1,500 \mathrm{~g}$ for $2 \mathrm{~min}$. Using the new 96 - well plates collecting filtrate (plasma). For each plate, add blank wells four randomly selected, two highlevel and two low-level QC control solutions respectively. Filtrate and QC solutions were all dried under $50^{\circ} \mathrm{C}$ pure nitrogen airflow. Derivatize the dried sample with $60 \mu \mathrm{L}$ acetyl chloride/1-butanol (10:90, v/v) mixture for $20 \mathrm{~min}$ at $65{ }^{\circ} \mathrm{C}$. After derivatization, as mentioned before, dried again for each sample. At last, dissolve each dried sample in $100 \mu \mathrm{L}$ fresh mobile phase solution for LC-MS/MS (Liquid Chromatograph Mass Spectrometer) analysis.

\section{LC-MS/MS metabolomic analysis}

We used the AB SCIEX 4000 QTrap (AB Sciex, 
Table 1 Demographic and clinical characteristics of patients

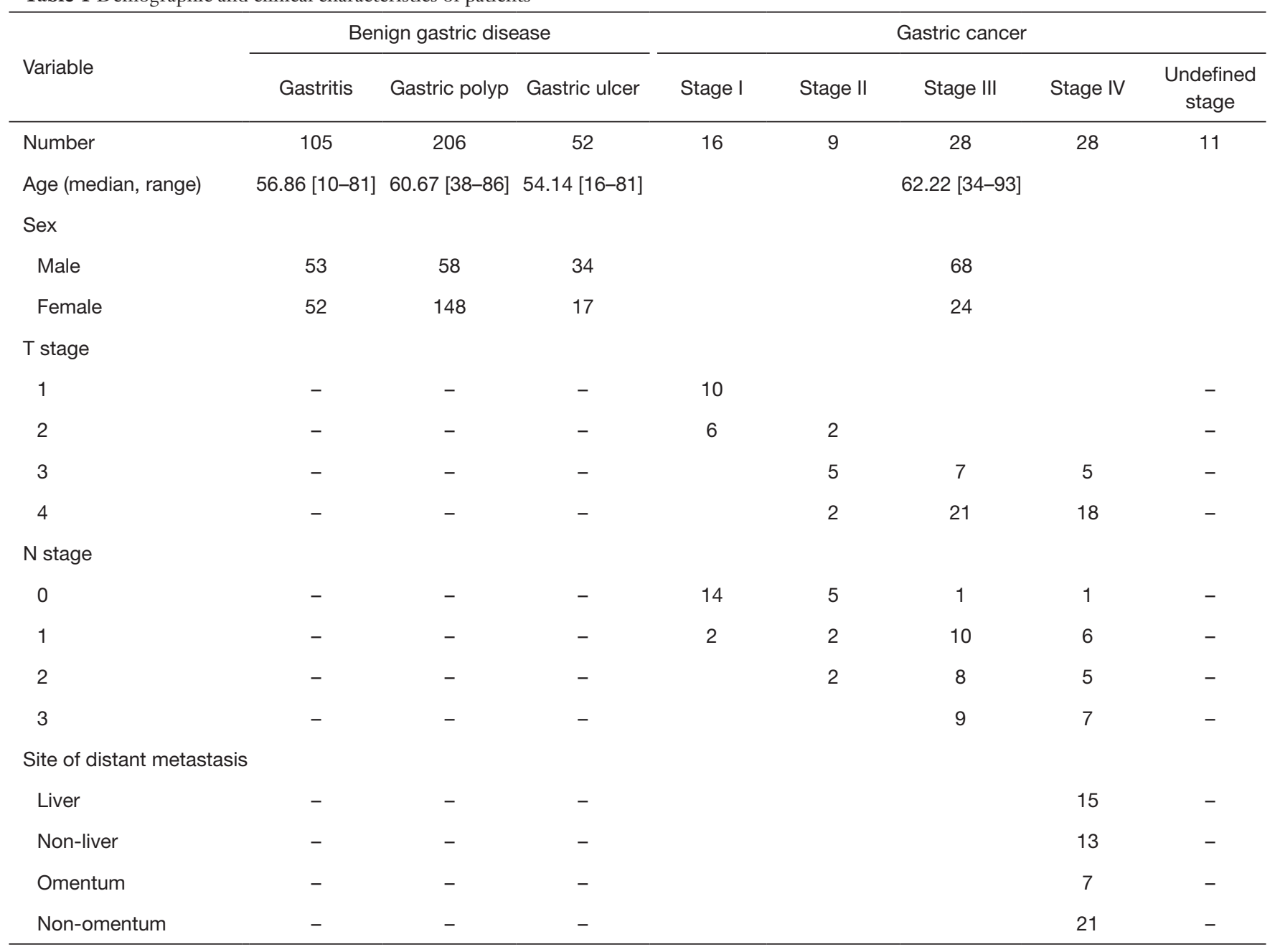

Stage of gastric cancer was based on the 8th edition of the American Joint Committee on Cancer (AJCC) Tumor-Node-Metastasis classification. T, tumor; N, node; M, metastasis.

Framingham, MA, USA) instrument to complete mass spectrometric analysis of the blood samples. For each run, every $20 \mu \mathrm{L}$ sample was injected. The mobile phase was $80 \%$ acetonitrile aqueous solution. Velocity of $0.2 \mathrm{~mL} / \mathrm{min}$ was set for initial flow rate. Subsequently, to adjust the flow rate to $0.01 \mathrm{~mL} / \mathrm{min}$ within $0.08 \mathrm{~min}$, remains constant until $1.5 \mathrm{~min}$, within $0.01 \mathrm{~min}$ returned to $0.2 \mathrm{~mL} / \mathrm{min}$, and held constant for $0.5 \mathrm{~min}$. Ion spray voltage of $4.5 \mathrm{kV}$ and curtain gas pressure was set to $20 \mathrm{psi}$. A pressure of 35 psi was set to ion source gas 1 and gas 2 . The auxiliary gas temperature was maintained at $350^{\circ} \mathrm{C}$.

Analyst v1.6.0 software (AB Sciex) was applied to MS data collection and system control. Chemo View 2.0.2 (AB Sciex) was used to preprocess the data. The MS ion source was an electrospray ionization source. Scan all analytes under positive mode. Parameters setting are shown in Table 2. According to different isotope standards, the software quantified the indicators of 23 amino acids.

\section{Statistical analysis}

The $t$-test was used to detect the normally distributed parameter variables between the gastric cancer group and the three benign groups, and the Wilcoxon rank test was used to detect the non-normally distributed variables. $\mathrm{P}<0.05$ was considered to be a significant difference. Venn diagrams were used to determine the shared different amino acids. Pearson's correlation coefficient was used to analyze the linear correlation between arginine and tumor markers, and analysis of variance was used to compare the difference 
Table 2 The amino acids detected, corresponding scan modes, equipment settings and quantification IS used in the study

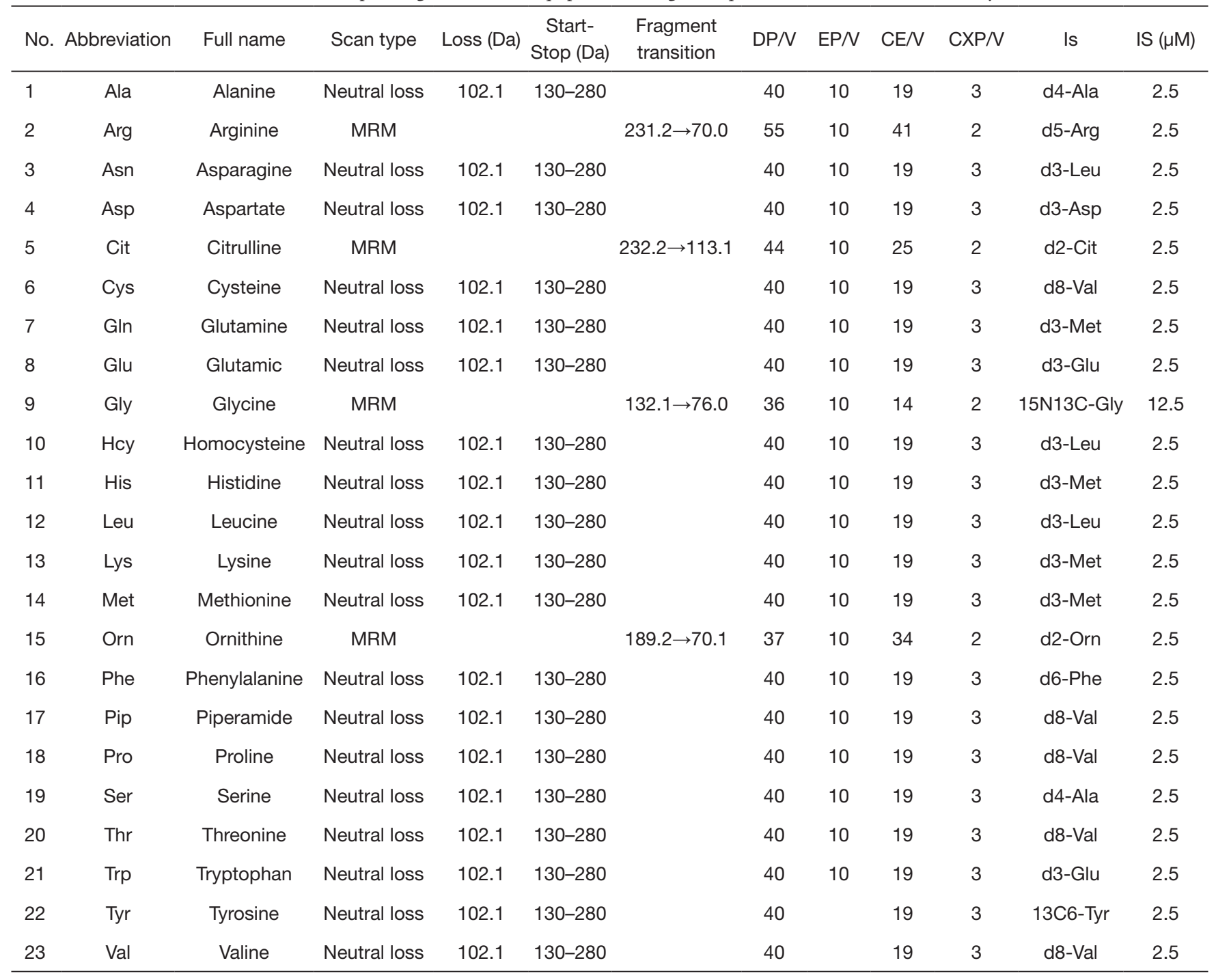

IS, internal standard; DP, declustering potential; EP, entrance potential; CE, collision energy; CXP, collision cell exit potential; MRM, multiple reaction monitoring.

in arginine expression in more than three groups (stage, differentiation, Lauren type). Follow-up of the patients were completed until the time of writing the manuscript or patients' death. Kaplan-Meier curves depict patient survival analysis, and Prism 8.0.2 was used to draw statistical analysis charts.

\section{Gene expression analysis}

To find the expression of the gene corresponding to the enzyme related to arginine expression in gastric cancer, we visited two online analysis platforms: http://cis.hku.
hk/TISIDB and http://gepia.cancer-pku.cn/index.html. The process was as follows. Click Expression DIY on the GEPIA platform, select Boxplot, enter gene names ASS1, ARG1, and ALS in turn, take 1 for $\log 2 \mathrm{FC}$, and 0.01 for $\mathrm{P}$ value, select gastric cancer, match the control group, and generate the difference in gene expression between gastric cancer and the control group. Click Survival, select Survival plot, enter ASS1, select gastric cancer, then OS and DFS (Disease-free survival)are selected successively to generate survival analysis graphs of the high-expression group versus the low-expression group. Enter ASS1 gene in the TISIDB platform and search for it. Click "Clinical" to select gastric 
Table 3 The differences of metabolites between GC patients and gastritis patients

\begin{tabular}{lccc}
\hline Variables & Gastric cancer group & Gastritis group & P value \\
\hline Glycine & 261.63 & 188.16 & $<.0001$ \\
Arginine & 14.1 & 9.06 & 0.0004 \\
Tryptophan & 51.1 & 43.68 & 0.0002 \\
Threonine & 30.74 & 26.69 & 0.0025 \\
Leucine & 132.33 & 115.29 & 0.0099 \\
Alanine & 129.41 & 151.15 & 0.0134 \\
Glutamate & 119.99 & 105.34 & 0.0117 \\
Citrulline & 25.58 & 21.37 & 0.0178 \\
Valine & 137.87 & 126.7 & 0.0442 \\
Asparagine & 74.88 & 66.79 & 0.0083 \\
\hline Shows & & &
\end{tabular}

Shows the amino acids with $\mathrm{P}<0.05$ between two groups.

cancer to generate the relationship between ASS1 and OS, staging and grading in gastric cancer.

\section{Gene oncology (GO) analysis}

Gastric cancer gene expression data were downloaded from the TCGA (The Cancer Genome Atlas) database and divided into two groups, namely, the high-expression and low-expression groups, according to the median value of ASS1. Limma R language packet was used to screen the differentially expressed genes between the two groups, and the FDR (false discovery rate) threshold value was set to 0.05 . The $\operatorname{LogFc}$ critical value was set as 2 to screen out differential genes. The top 10 biological functions of differential gene enrichment between the two groups were selected to plot the BARPLOT for GO enrichment analysis. The biological function was changed by the highexpression group of $A S S 1$, and the GO enrichment analysis chord diagram was drawn.

\section{Results}

\section{Significant differences in metabolites between gastric cancer and other groups}

In this study, 454 patients were enrolled and divided into four groups: 92 patients in the gastric cancer group, with an average age of 62.22 years (range, 34-93 years); 51 patients in the gastric ulcer group, with an average age of 60.67 years (range, 38-86 years); 206 patients in the gastric polyp group had an average age of 54.14 years (range, 16-87 years); 105 patients in the gastritis group, with an average age of 56.86 years (range, 10-81 years). In terms of age and body mass index, there was no statistical difference between the gastric cancer group and the other three groups, which were comparable. The indicators of different metabolites in the gastric cancer group were compared with those in the other three groups, and the results obtained are shown in Tables 3-5.

\section{Shared differential metabolites}

As shown in Figure 2A, we focused on the differential metabolites of gastric cancer $v s$. gastritis, gastric cancer $v s$. gastric ulcer, gastric cancer $v$ s. gastric polyp. The differences between three pairs of groups showed a certain overlap, the differential metabolites intersected, and finally arginine appeared in the common intersection of the three groups. The box diagram of arginine is shown in Figure $2 B$.

\section{Relationship between arginine and prognosis}

The gastric cancer group was further stratified according to clinicopathological parameters (e.g., TNM stage, Lauren type, degree of differentiation, whether the tumor markers were elevated, etc.), and the relationship between arginine and these parameters was established. We found that arginine levels in nonmetastatic gastric cancer (stages I, II, and III) were 
Table 4 The differences of metabolites between GC patients and gastric polyp patients

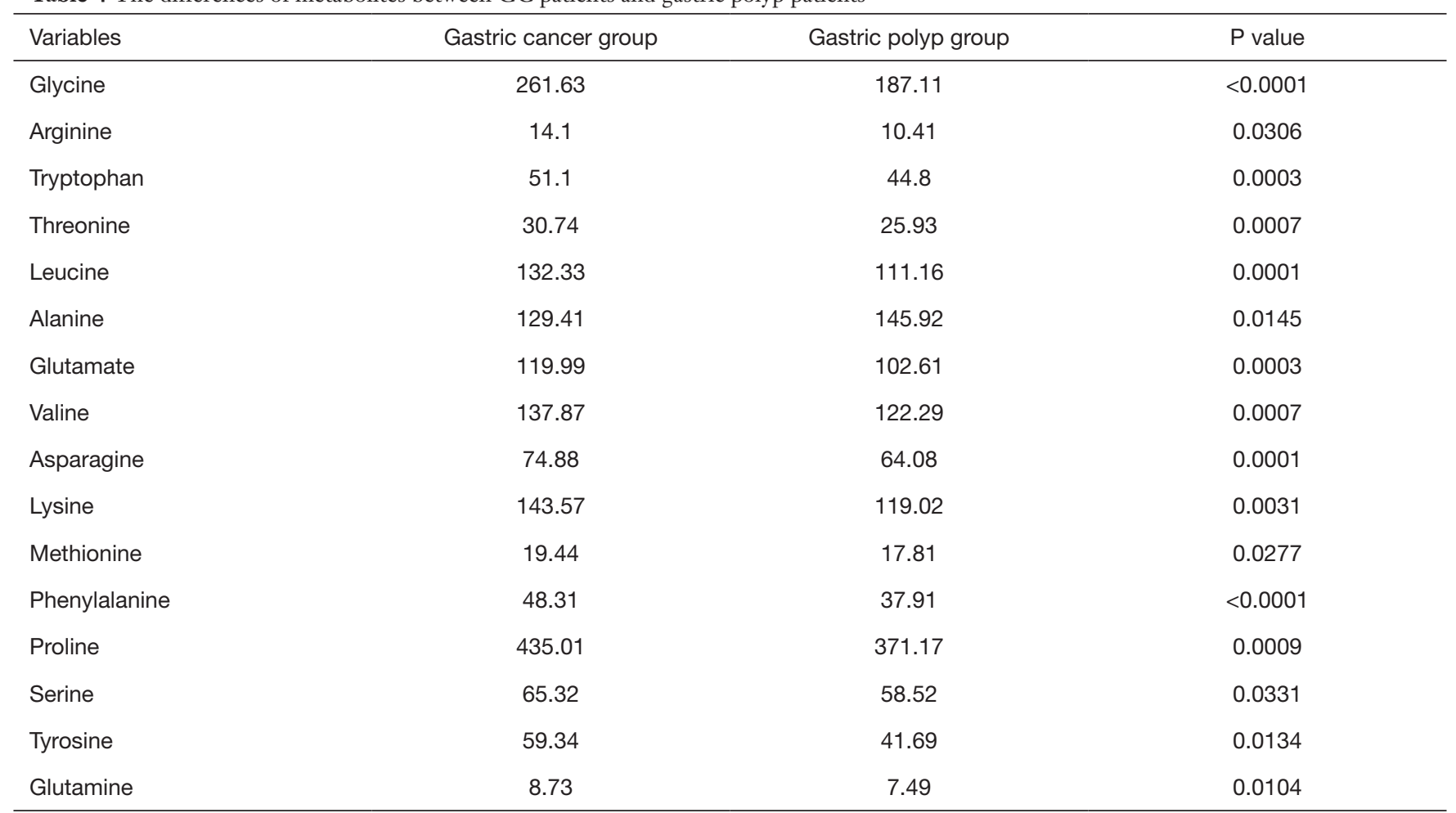

Shows the amino acids with $\mathrm{P}<0.05$ between two groups.

Table 5 The differences of metabolites between GC patients and gastric ulcer patients

\begin{tabular}{lccc}
\hline Variables & Gastric cancer group & Gastric ulcer group & $P$ value \\
\hline Arginine & 14.1 & 9.85 & 0.0065 \\
Lysine & 143.57 & 114.59 & 0.0226 \\
\hline
\end{tabular}

Shows the amino acids with $\mathrm{P}<0.05$ between two groups.

significantly higher than those in metastatic gastric cancer (stage IV) $(\mathrm{P}=0.0013)$. In patients with stage IV gastric cancer, we compared the blood arginine levels between liver metastasis and non-liver/peritoneal metastasis and non-peritoneal metastasis, and the results showed no significant difference. In the comparison with other subgroups, although the $\mathrm{P}$ value was not statistically significant, it also showed a certain trend (Figure 3A,B,C,D,E,F, Table 6). According to the KaplanMeier survival curve of the OS of the follow-up patients, due to changes in the addresses and contact information of some patients, 10 patients were lost to follow-up. KaplanMeier survival curves of two groups of patients with GC defined based on an arginine level cutoff value of 10.895 (median). The two groups showed perfect separation, with a $\mathrm{P}$ value for the log-rank test of 0.0003 , HR for death was $0.4030,95 \%$ CI (0.2387-0.6806) (Figure 3G).

\section{Correlation analysis between arginine and gastric cancer tumor markers}

We analyzed the correlation between blood levels of arginine and gastric cancer tumor markers CEA, CA19-9 and CA72-4. We found that arginine negatively correlated with CEA and CA199, and the Pearson correlation coefficients were -0.14 and -0.12 , respectively. Figure 4 is a 
A

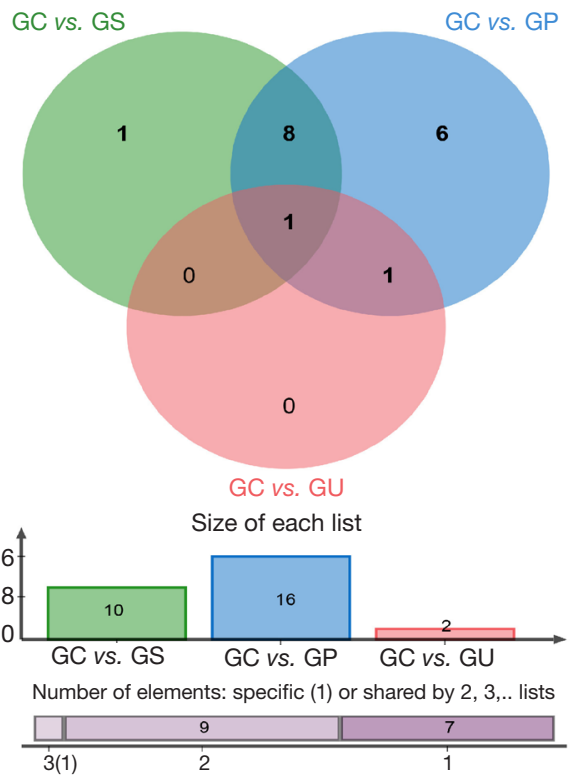

B

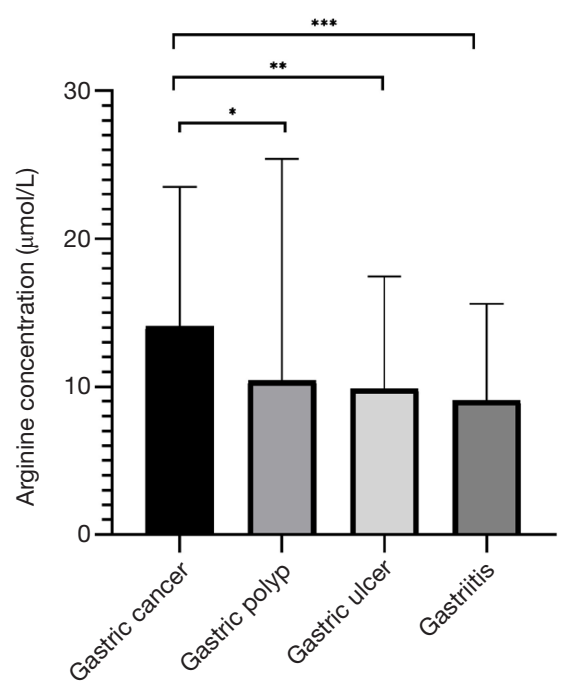

Figure 2 The screening of arginine and its level in each group. (A) Venn-diagram showing markers shared between pairs of groups; (B) the difference of blood arginine concentration between gastric cancer group and gastric polyp, gastric ulcer, and gastritis group. ${ }^{*}, \mathrm{P}<0.05 ;{ }^{* *}$, $\mathrm{P}<0.01 ;{ }^{* * *}, \mathrm{P}<0.001$.

heat map that reflects this relationship.

\section{Gene expression of arginine metabolic enzymes}

Arginine is involved in the urea cycle, and the circulation pathway is shown in Figure 5 A. In the metabolic pathway, the ASS1 and ALS enzymes are involved in the synthesis of arginine, and Arg1 catalyzes the decomposition of arginine. ASS1 is a key metabolic enzyme in the urea cycle. As shown in Figure 5B, gastric cancer gene analysis in the GEPIA online platform showed that, compared with the control group, elevated expression of ASS1 was significantly different in gastric cancer tissues, while the expression of $A L S$ and $A r g 1$ was not significantly different. The high expression of $A S S 1$ in gastric cancer may be the mechanism of the elevated arginine levels in gastric cancer patients.

\section{Relationship between metabolic patbway key enzyme ASS1 and prognosis in patients with gastric cancer}

Based on the relationship between arginine and clinicopathological parameters, and the relationship between arginine metabolism key enzyme ASS1 and arginine, we attempted to find the relationship between ASS1 and the prognosis of gastric cancer patients in the TISIDB platform. The results were different from many tumor types. High expression of ASS1 in gastric cancer can extend the patient's survival. The Kaplan-Meier plot shown in Figure $6 \mathrm{~A}$ compares the OS of gastric cancer patients with high expression of ASS1 with those with low expression of ASS1. Patients with 10 years of followup had a longer survival in the group with high expression of ASS1, with a P value of 0.0445 , which was statistically significant. We also performed a survival analysis of ASS1 in another gene pool GEPIA, and found that the DFS of gastric cancer patients in the group with high expression of ASS1 was also significantly prolonged compared with the group with low expression, as shown in Figure 6B. We continued to explore the relationship between ASS1 and clinicopathological parameters such as gastric cancer stage and differentiation, and the results showed that ASS1 did not show good separation in terms of stage, but the higher the degree of differentiation, the higher the expression of ASS1. The difference of Grade 1, Grade 2 and Grade 3 was statistically significant $(\mathrm{P}<0.0001)$. The results are shown in Figure $6 C$ and $D$.

\section{GO analysis}

Figure 7 shows the results of GO enrichment analysis. The barplot Figure $7 A$ shows the differences in the genetic 

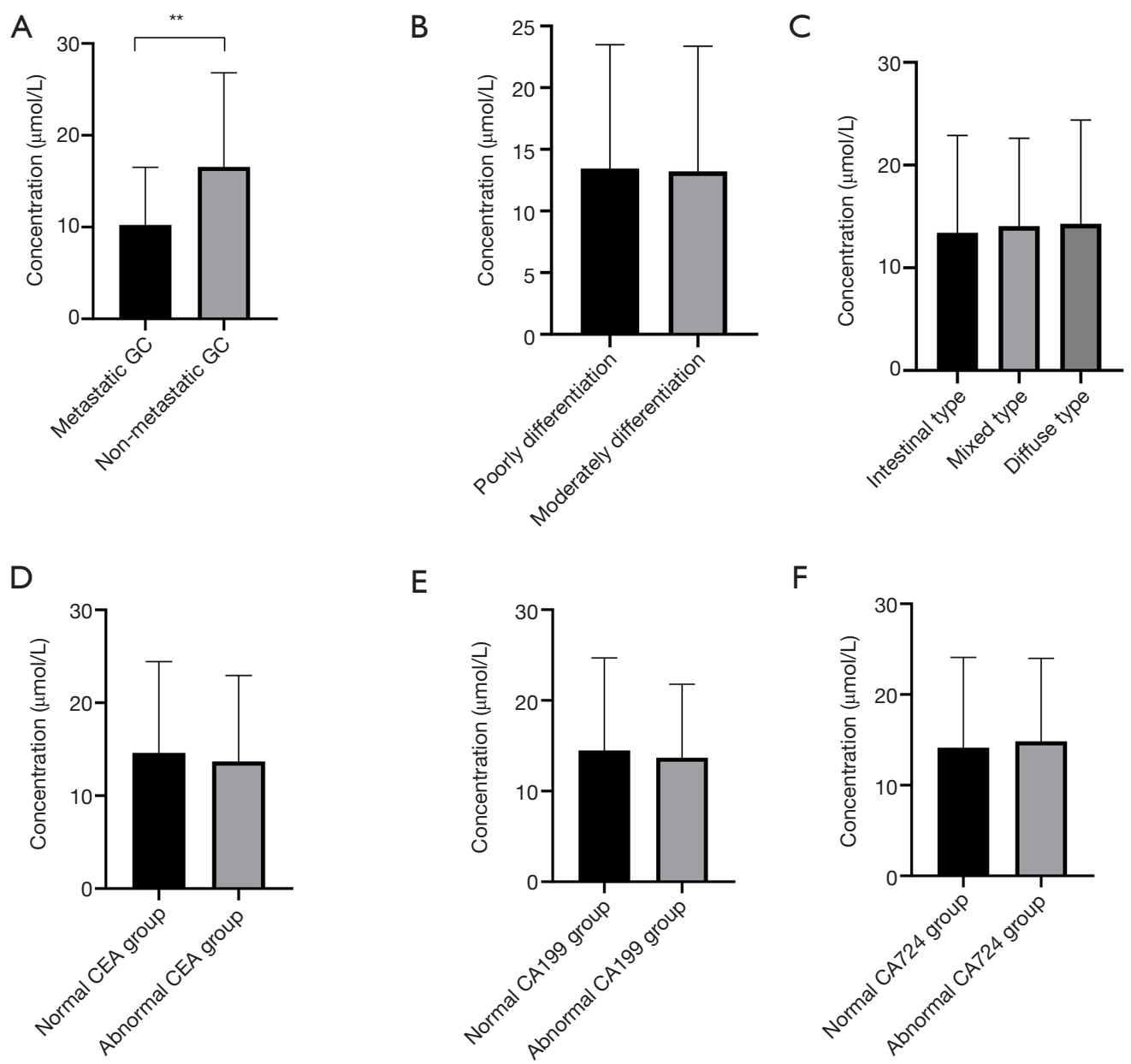

$\mathrm{E}$

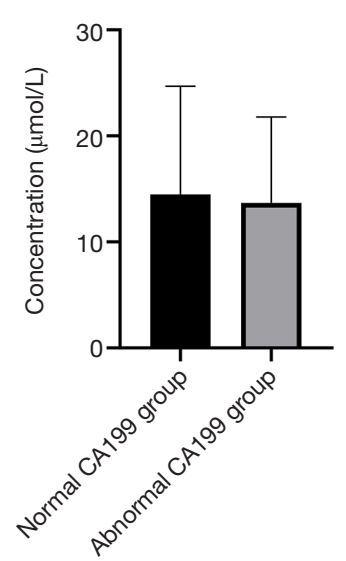

$\mathrm{F}$
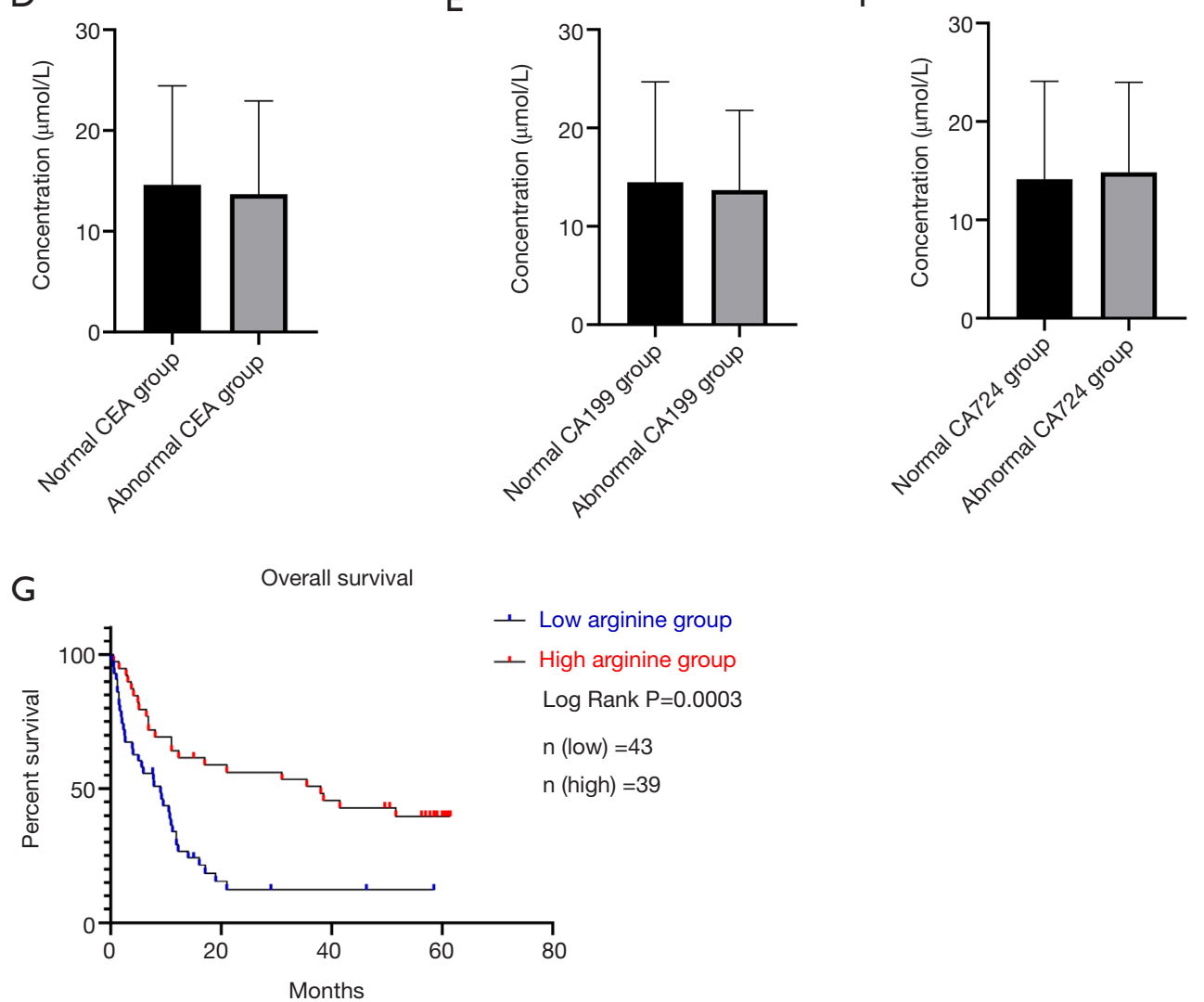

Figure 3 Plasma arginine level in human GC is correlated with patient prognosis. (A) The difference in arginine level is compared according to whether there is distant metastasis $(\mathrm{P}=0.0013, \mathrm{M} 0$ vs. $\mathrm{M} 1)$. (B,C,D,E,F) Compared with the difference in arginine levels according to differentiation, Lauren classification, CEA normal or abnormal, CA19-9 normal or abnormal, CA72-4 normal or abnormal respectively, the P value was not statistically significant. (G) Kaplan-Meier survival curves of two groups of patients with GC defined based on an arginine level cutoff value of 10.895 (median). The survival rate of the high level group (39 cases) was significantly better than that of the low level group (43 cases). Log rank $\mathrm{P}=0.0003$. ** $\mathrm{P}<0.01$. 
Table 6 Relationship between arginine and prognostic variables

\begin{tabular}{|c|c|c|c|}
\hline Prognostic variables & Samples & Arginine level ( $\mu \mathrm{mol} / \mathrm{L})$ & $P$ value \\
\hline $1-2$ & 18 & $14.05 \pm 9.23$ & 0.83 \\
\hline $3-4$ & 57 & $14.61 \pm 9.64$ & \\
\hline \multicolumn{4}{|l|}{$\mathrm{N}$ stage } \\
\hline $2-3$ & 31 & $16.41 \pm 11.41$ & \\
\hline \multicolumn{4}{|l|}{ M stage } \\
\hline 0 & 53 & $16.37 \pm 10.17$ & $0.0013^{*}$ \\
\hline 1 & 28 & $10.24 \pm 6.15$ & \\
\hline Non-liver metastasis & 13 & $10.56 \pm 6.57$ & \\
\hline Omentum metastasis & 7 & $11.47 \pm 5.22$ & 0.53 \\
\hline Non-Omentum metastasis & 21 & $9.83 \pm 6.38$ & \\
\hline \multicolumn{4}{|l|}{ Differentiation } \\
\hline Poorly differentiated & 28 & $13.46 \pm 9.85$ & 0.88 \\
\hline Moderately differentiated & 12 & $14.01 \pm 10.35$ & \\
\hline \multicolumn{4}{|l|}{ Lauren type } \\
\hline Intestinal & 20 & $13.42 \pm 9.23$ & 0.95 \\
\hline \multicolumn{4}{|l|}{ CA19-9 } \\
\hline Normal & 63 & $14.5 \pm 10.1$ & 0.72 \\
\hline Abnormal & 20 & $13.68 \pm 7.89$ & \\
\hline \multicolumn{4}{|l|}{ CA72-4 } \\
\hline Normal & 55 & $14.13 \pm 9.86$ & 0.75 \\
\hline Abnormal & 28 & $14.83 \pm 8.99$ & \\
\hline
\end{tabular}

${ }^{*}$, represents a significant difference. Mean \pm SD was used to express arginine value.

effect in the ASS1 high- and low-expression groups of the top 10 biological processes, such as the humoral immune response, stable gastrointestinal epithelium, epithelial maintenance structures and cell adhesion, etc., the six types of molecular functions such as receptor ligand activity, chemokine activity, etc., and the locations in the cell. Figure $7 B$ is a string diagram of the GO enrichment analysis, showing that each differential gene in the ASS1 high-expression group has its own role in the metabolic pathway, affecting the above 10 biological functions. 


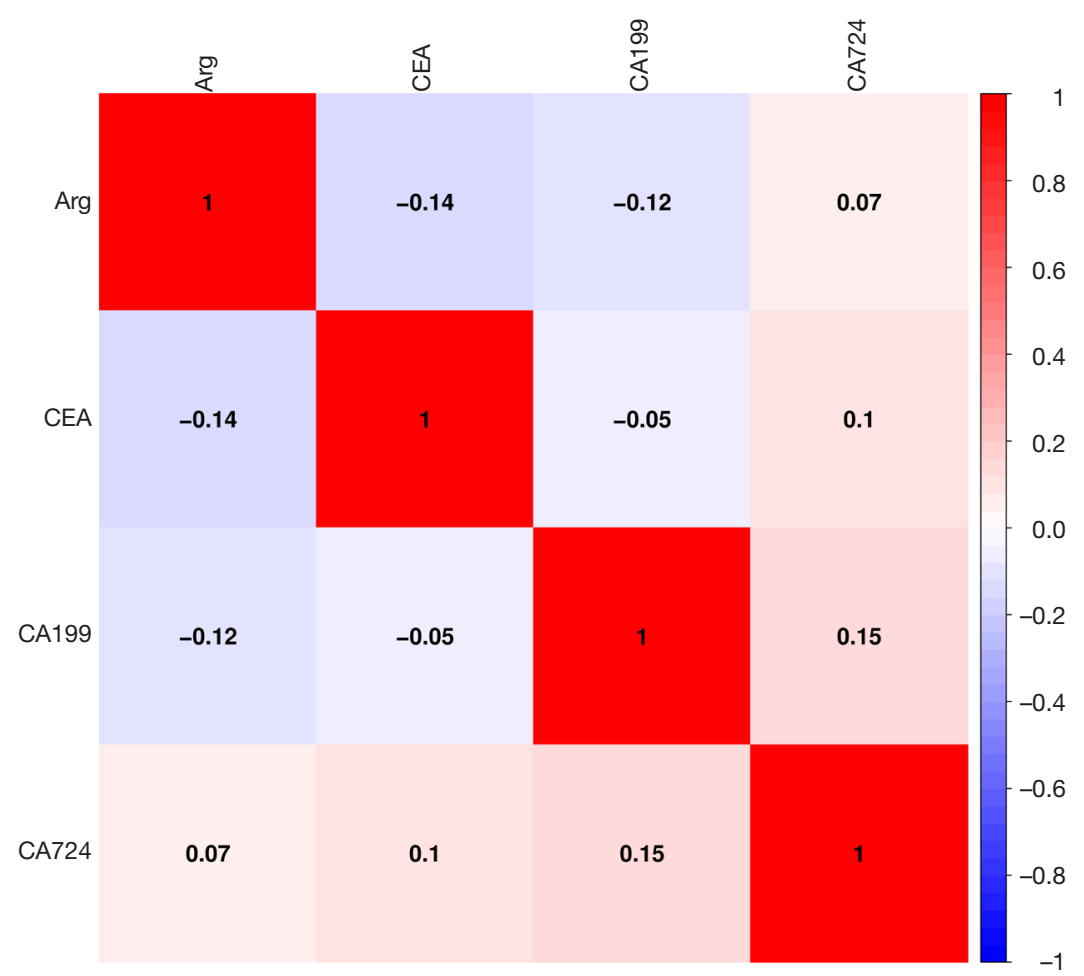

Figure 4 Results of correlation analysis between arginine and tumor markers. The value represents the Pearson correlation coefficient. Among them, the correlation coefficients between arginine and CEA and CA19-9 are -0.14 and -0.12 , respectively, and there is a certain linear negative correlation.

In general, as shown in the Figure $7 B$, when $A S S 1$ is highly expressed, the overall effect of the different genes enhances the immune response, cell adhesion and other biological functions.

\section{Discussion}

Gastric cancer is characterized by a slow development process from precancerous lesion to malignant stages. In the different stages of disease development, the level of metabolites differs, affecting the prognosis. A number of studies have reported a spectrum of differential expression of metabolites between gastric cancer and gastric benign diseases (4-8). In this study, benign gastric diseases such as atrophic gastritis, gastric polyps and gastric ulcers were included, all of which are precancerous lesions of gastric cancer.

Metabolomics aims at qualitative and quantitative analyses of metabolites in organisms. In recent years, great breakthroughs have been made in the fields of cancer diagnosis, analysis of antitumor therapy and metabolic targeted therapy $(13,14)$, because the metabolites in the body are the result of the joint action of heredity (gene, transcription) and environment, which makes the metabolomics method simple and intuitive (15). In recent years, more and more studies in the field of metabolomics have been devoted to finding markers that can assist clinicians in understanding the etiology, pathogenesis, diagnosis, evaluation of treatment response and treatment decision-making of gastric cancer (16-18).

Amino acids are present in almost all cells and are basic components of cells. Metabolic imbalance is a key factor in the occurrence and development of tumors (3). Therefore, in the existing metabolomic studies, amino acid metabolism profiles provide important information for researchers to understand the pathogenesis of tumors at the molecular level. Arginine is a non-essential amino acid that is required by both normal and tumor cells for basic biological processes such as protein synthesis and polyamine synthesis. Therefore, arginine plays an important role in the development of tumors. Hu et al. reported that arginine is related to the molecular typing of breast cancer (11), and Selvi et al. reported that arginine and its metabolites 
A
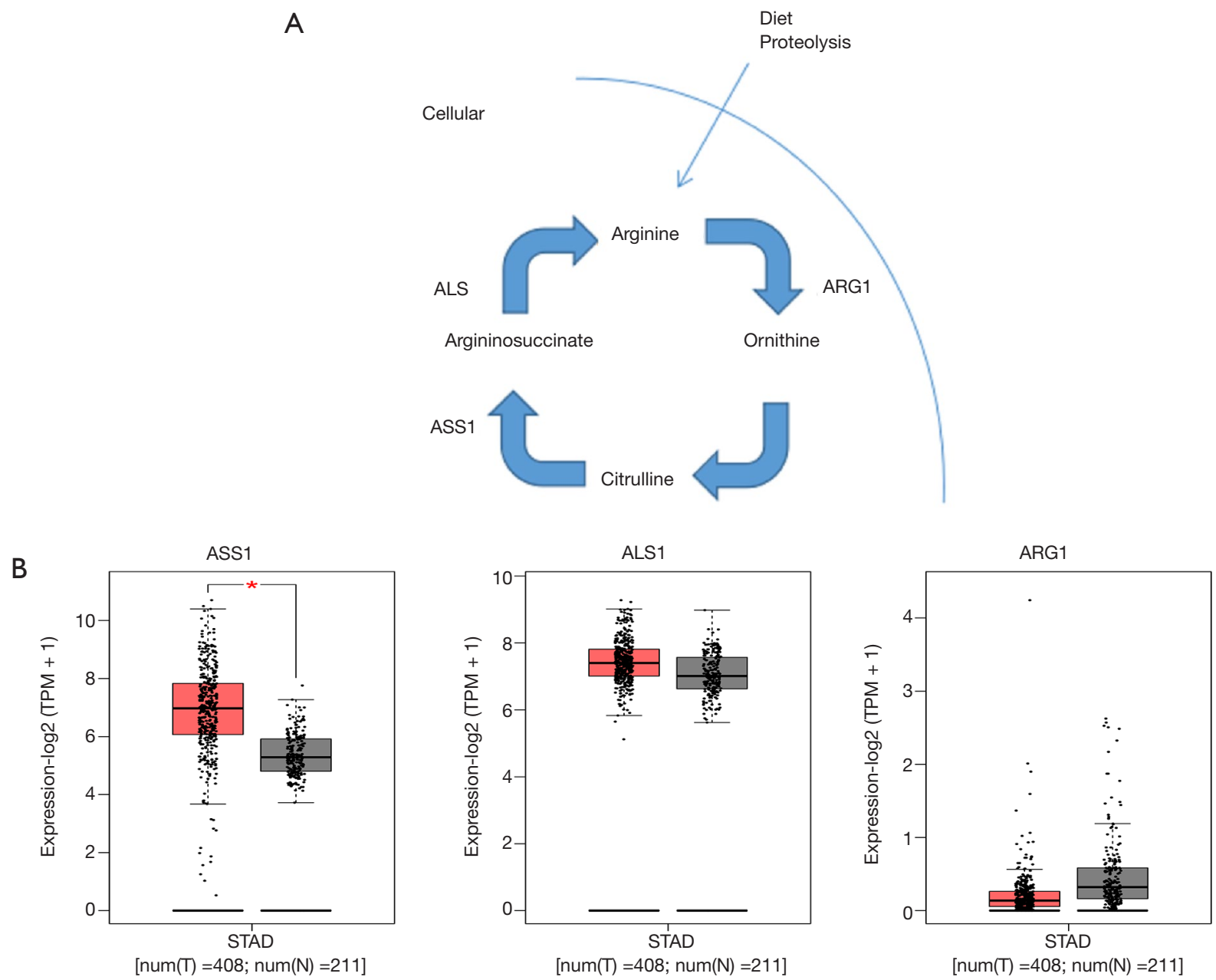

Figure 5 The elevated expression of the ASS1 gene in gastric cancer may cause changes in the blood arginine level of the patient. (A) Metabolic pathways of arginine; (B) difference in expression of mRNA of ASS1 gene, ALS1 gene and ARG1 gene between gastric cancer tissue and normal tissue. *, $\mathrm{P}<0.05$. ASS1, Argininosuccinate Synthase 1; ALS, argininosuccinate lyase; ARG1, arginase; T represents tumor tissue; $\mathrm{N}$ represents normal tissue.

can assist the diagnosis of prostate cancer (10). In other tumors, arginine levels are generally decreased in the cancer group. Arginine metabolism disorder has an impact on the quantity and function of T cells (19). If arginine is lacking, the effect of the interaction between $\mathrm{T}$ cells and tumor antigens will be greatly reduced $(20,21)$, influencing the immune system (22). Moreover, the absence of arginine exacerbates the infection of host cells by Helicobacter pylori, which leads to the occurrence and development of gastric cancer (23), increasing gastric carcinogenesis (24). However, in the field of gastric cancer research, Zhang et al. found that the arginine level of mice in the gastric cancer group was significantly higher than that in the healthy control group (12). Jung et al. found that the arginine index of gastric cancer patients was higher than that of normal subjects according to the urinary metabolic profile (16). In our study, LC-MS/MS was used to quantitatively analyze the levels of 23 amino acids in the plasma of 454 enrolled patients. The result showed significant differences in blood amino acid levels between the gastric cancer group and the benign gastric disease group (gastritis, gastric ulcers, gastric polyps). We concluded there is also abnormal metabolism of arginine in patients with gastric cancer. Arginine was the common differential amino acid between gastric cancer and the other three benign disease groups. The index of arginine was higher than that in each group of benign 
A

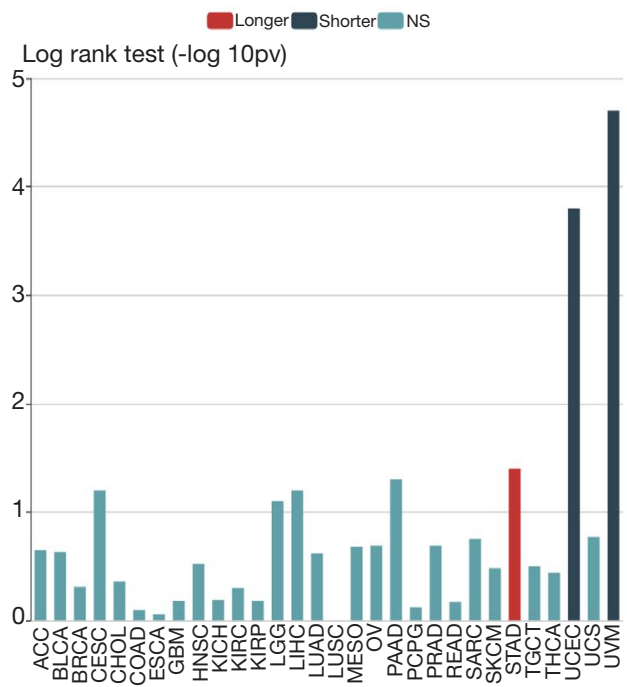

B

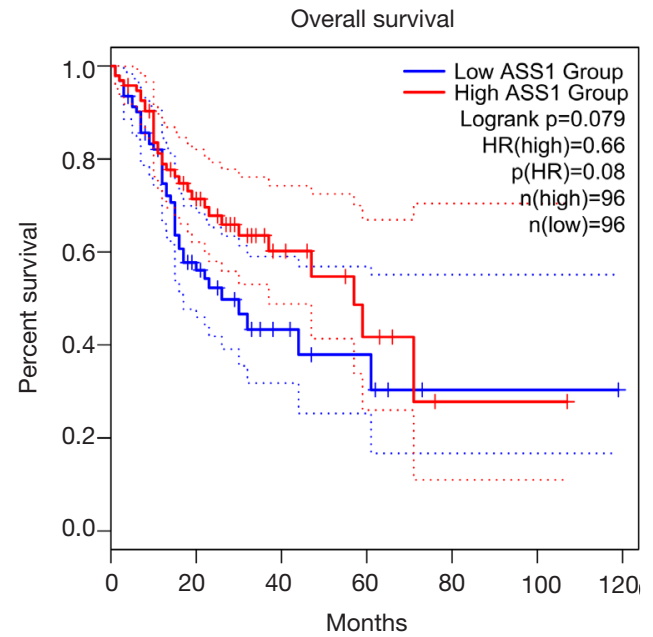

C

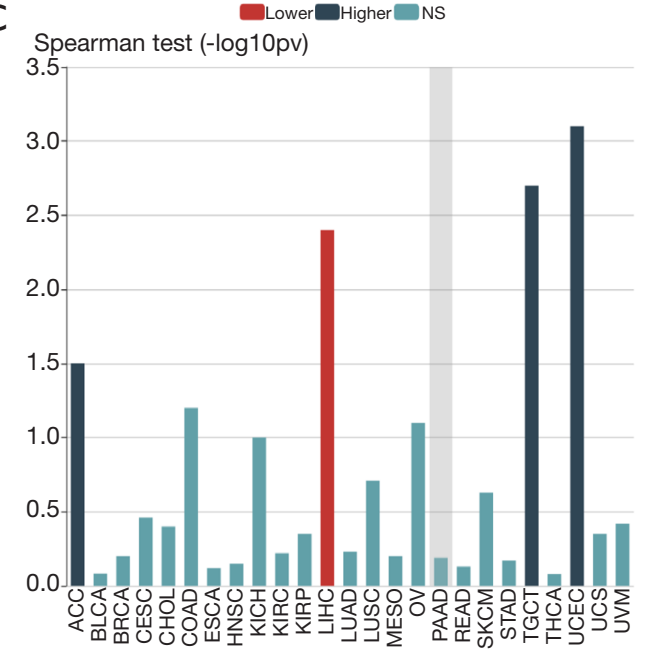

STAD :: ASS1_exp
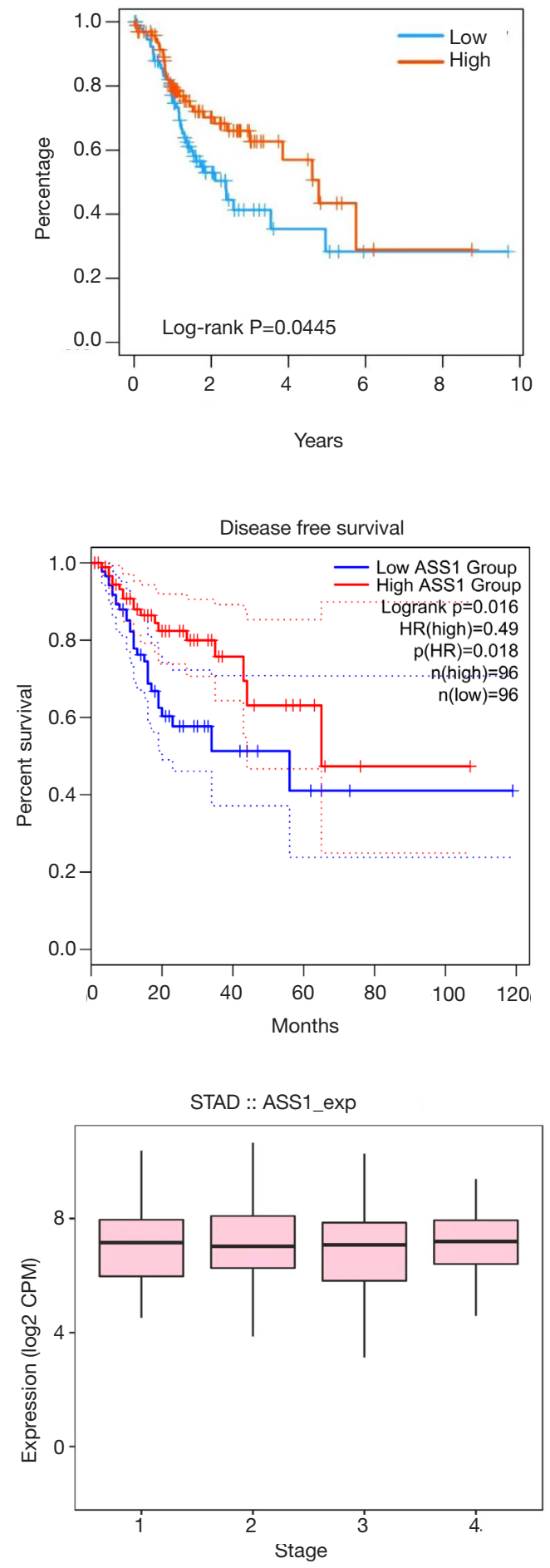

Spearman: rho $=-0.021, \mathrm{P}=0.674$ 

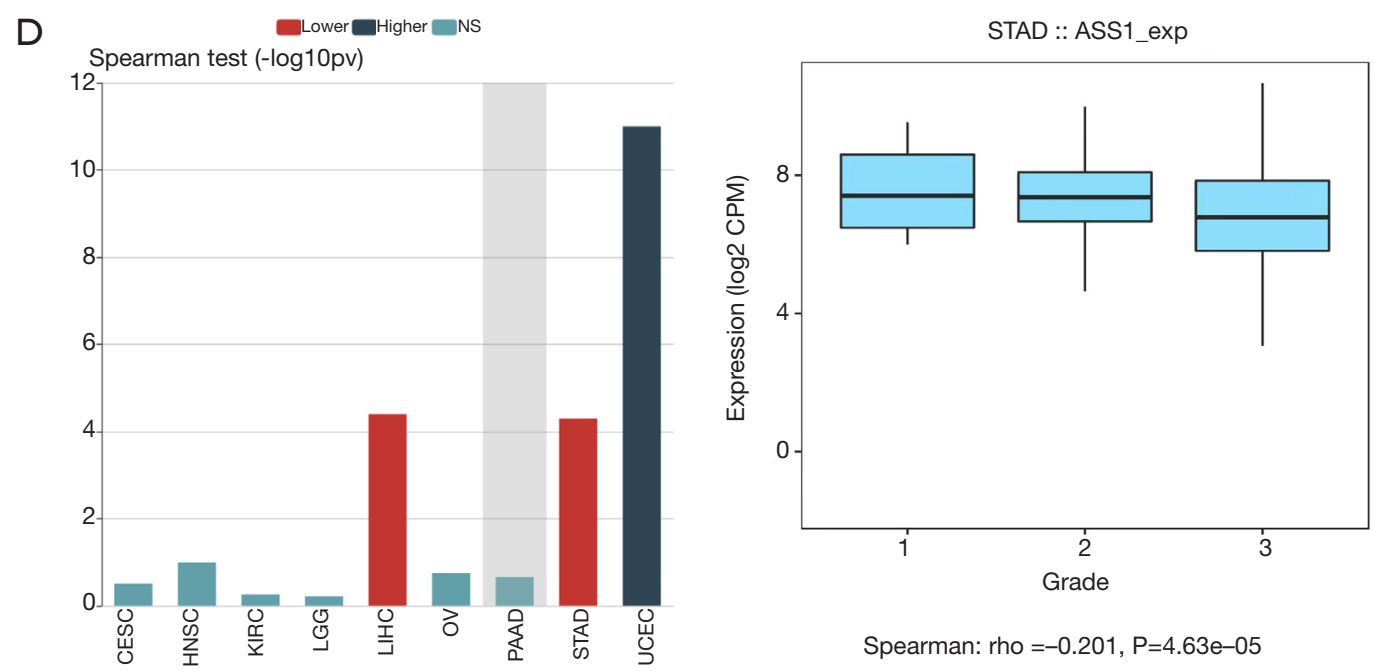

Figure 6 ASS1 gene expression is also related to the survival of patients with gastric cancer. (A) TISIDB online analysis platform shows that ASS1 gene expression affects OS in patients with gastric cancer. ASS1 gene expression is beneficial to the survival of patients with gastric cancer. Kaplan-Meier survival curves of two groups of patients with GC based on an ASS1 expression (median). The 10-yr survival rate of the higher ASS1 expression groups was significantly greater than that of the lower expression groups (log-rank P =0.0445). (B) GEPIA online analysis platform shows that ASS1 gene expression affects OS and DFS in gastric cancer patients. The data analysis platform included 192 patients with operable gastric cancer, which were divided into high expression group and low expression group according to the median of ASS1.After 10 years of patient follow-up, the OS and DFS of the higher ASS1 expression groups were better than that of the lower expression groups. The data of DFS is statistically significant (log-rank $\mathrm{P}=0.016)$. (C) TISIDB online analysis platform shows that there is no statistically significant between ASS1 gene expression and gastric cancer staging. (D) TISIDB online analysis platform shows that ASS1 gene expression is related to histological grade of gastric cancer. $(\mathrm{P}<0.001$; grade1 vs. grade 2 vs. grade 3$)$.

gastric diseases.

Previous studies have reported that arginine is related to the T stage of gastric cancer $(16,25,26)$. We analyzed the relationship between arginine and clinicopathological parameters such as differentiation and staging of gastric cancer. Although there was no increasing or decreasing trend of arginine in the different stages, we found that the arginine index in patients with non-metastatic gastric cancer (stages I, II and III) was higher than that in patients with metastatic gastric cancer (stage IV), and was statistically significant. The level of blood arginine in patients with moderately differentiated gastric cancer was higher than that in patients with poorly differentiated gastric cancer, and the level of blood arginine in patients with tumor markers CEA and CA19-9 in the normal range was higher than that in patients with abnormal tumor signs, although these were not statistically significant, which may be related to insufficient numbers of cases. Further grouping of patients in stage IV showed that gastric cancer patients with abnormal arginine metabolism did not show a tendency of directional metastasis, which may also be related to the insufficient number of cases after grouping. At present, tumor markers are often used to evaluate the prognosis of patients and dynamically monitor tumor patients. Arginine negatively correlated with tumor markers CEA, and CA199. These results suggested that the prognosis of gastric cancer with elevated arginine level is relatively good. According to Shu et al., arginine regulates gastric cancer cell apoptosis by reducing the expression of anti-apoptotic protein Bcl-2 and protecting and increasing apoptotic protein 553 (27). Other studies have reported that arginine inhibits the growth of gastric cancer cell lines by increasing the expression of caspas8 (28). This may be the mechanism by which arginine plays an antitumor role in patients with gastric cancer. Therefore, gastric cancer patients with high arginine may have a better prognosis, so we followed up the patients with gastric cancer to establish the relationship between arginine and OS. Unfortunately, 10 patients were lost to follow-up. The OS of gastric cancer patients with high expression of arginine was significantly longer than that of patients with low expression of arginine. Arginine can be used as a prognostic parameter for patients with 
A

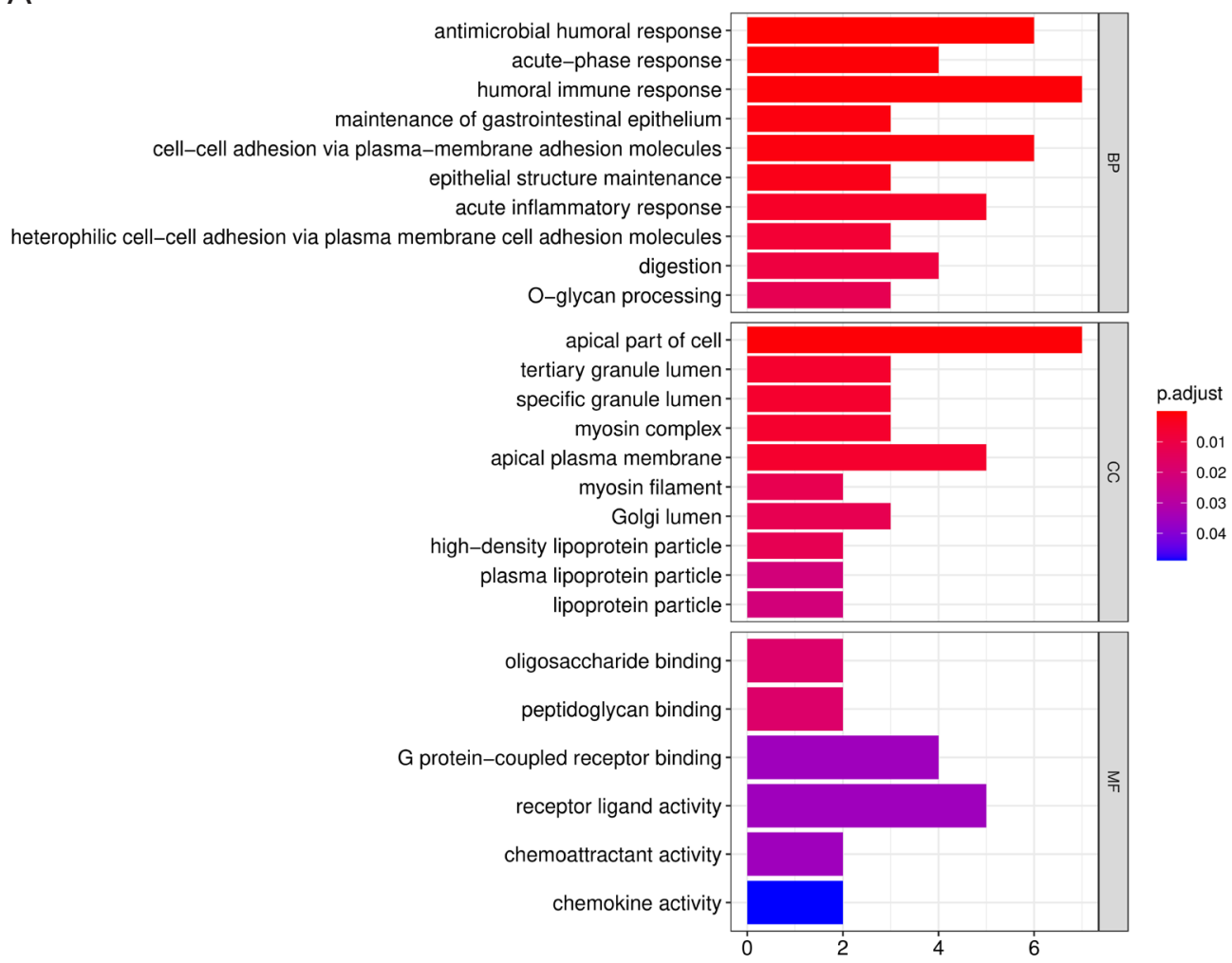

B

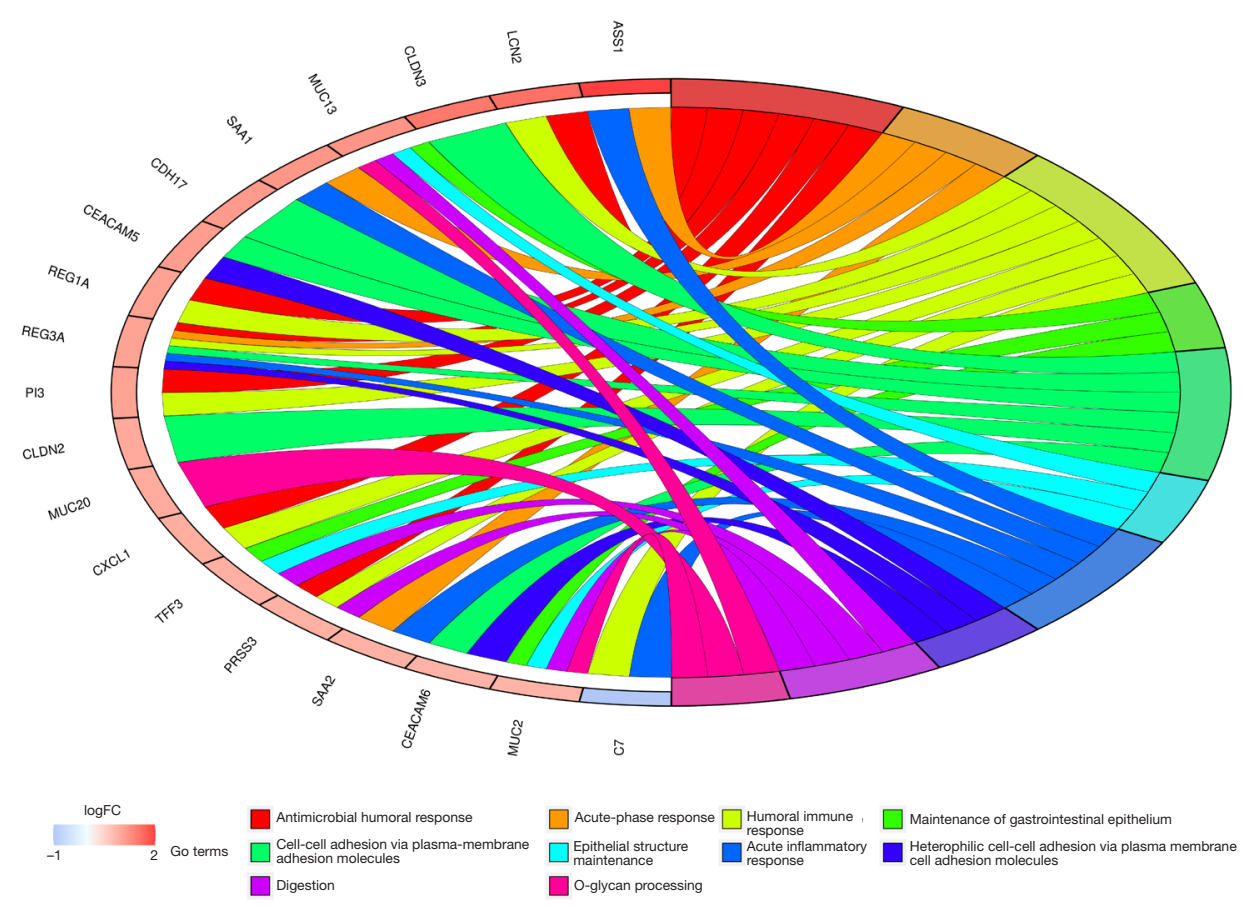

Figure 7 The biological process corresponding to the high expression of ASS1 gene by GO analysis. (A) Bar plot of GO enrichment analysis; (B) Chord graph of GO enrichment analysis. 
gastric cancer.

Through in-depth mechanism analysis, we investigated why arginine is overexpressed in the blood of gastric cancer patients. Arginine participates in the urea cycle, and the key metabolic enzyme regulating the production of arginine is ASS1. Overexpression of ASS1 in gastric cancer was statistically significant, but there was no significant difference in the expression of genes corresponding to other enzymes in the pathway. This may be the reason for the increased arginine level in patients with gastric cancer. ASS 1 catalyzes citrulline and aspartic acid to form arginine succinic acid and arginine under the action of ASL. Research on ASS1 in the tumor field has been reported (29), and the expression of ASS1mRNA is increased in ovarian cancer and colon cancer, while decreased in melanoma, hepatocellular carcinoma, mesothelioma, renal cell carcinoma and prostate cancer (30). Studies have shown that ASS1 inhibits the metastasis of hepatocellular carcinoma (31), and that ASS1 inhibits the invasion and metastasis of breast cancer cells (32). Bateman et al. reported that pharmacological inhibition or genetic ablation of ASS1 impairs colorectal cancer pathogenicity (33). In ovarian cancer, the promoter DNA methylation of ASS1 is regulated by epigenetics, and the expression of $A S S 1$ is silenced, which leads to cisplatin tolerance. The lower the expression of $A S S 1$, the worse the prognosis of ovarian cancer $(34,35)$. It can be seen that the prognostic effect of ASS1 expression differs among tumors.

Based on the relationship between arginine and the prognosis of gastric cancer, the relationship between ASS1 enzyme and arginine, and the effect of $A S S 1$ expression on the prognosis of other tumors, and through the analysis of gastric cancer data in TCGA database, we found that the expression of $A S S 1$ also affected the prognosis of patients with gastric cancer, and that the OS and DFS were significantly prolonged in the group with high expression of ASS1. Further analysis of the relationship between ASS1 and differentiation and staging showed that although there was no trend in each stage, we found good differentiation and high expression of ASS1 in gastric cancer in the stratification of histological grade, which was consistent with a longer survival period of patients with high expression of $A S S 1$.

In order to analyze the mechanism of good DFS and OS in the ASS1 high-expression group, GO enrichment analysis was performed. Because gene regulation is a complete system, it is not possible to analyze only one gene or an isolated metabolic pathway. Therefore, the role of different genes in the ASS1 high- and low-expression groups in gastric cancer was investigated from the aspects of biological process, cell composition and molecular function. We found that the strongest relationship with the biological function of the different genes was the stability of gastrointestinal epithelium, maintenance of epithelial structure, humoral immune response and so on. Based on the overall effect, the high expression of ASS1 enhances the stability of the gastrointestinal epithelium and the maintenance of epithelial structure, so if the expression of ASS1 is high, the degree of differentiation of cancer tissue is better in patients with gastric cancer. Through our analysis of clinical data, we found that the level of arginine in nonmetastatic gastric cancer was significantly higher than that in metastatic gastric cancer, which may be related to the stability of the gastrointestinal epithelium and enhanced cell adhesion in the ASS1 high-expression group. In addition, the high expression of $A S S 1$ also enhanced the humoral immune response, which may be the mechanism of the good DFS and OS in the high-expression group.

Some tumors with low expression of $A S S 1$, can be described as arginine nutritionally deficient tumors. In recent years, some researchers have proposed arginine targeted therapy for such tumors. Because these tumors cannot synthesize arginine themselves, they rely on exogenous arginine to promote the growth of tumor cells, so the degradation of exogenous arginine can promote the apoptosis of tumor cells. Arginine deiminase (DI-PEG20) is clinically used to activate the Bax signaling pathway of ASS1-methylated cells and induce cell apoptosis. This method has achieved good therapeutic effects in argininedeficient tumors such as melanoma and liver cancer $(36,37)$. Different from those tumors, ASS1 is mostly highly expressed in gastric cancer patients (38), so there is still a lack of research on arginine targeted therapy. Because arginine plays an antitumor role, and gastric cancer patients with high expression of ASS1 have better OS and DFS, increasing the level of ASS1 or arginine may benefit gastric cancer patients. It has been reported that an arginine-rich diet during the preoperative period is beneficial to the recovery of patients, reduces postoperative complications and shortens the length of hospital stay with gastric cancer patients (39). A randomized controlled trial shows that the postoperative use of enteral nutrition enriched with arginine may have a significant reduction in the risk of death during the early period after surgery (first 6 months) in stage IV gastric cancer patients (40). There are still some limitations in the current study. We did not verify the ASS1 
gene or ASS1 protein data in the included gastric cancer patients, but only obtained it in the TCGA database. In future studies, we will expand the sample size of patients and focus on arginine targeted therapy for gastric cancer to explore whether postoperative adjuvant exogenous arginine supplementation can improve the OS of gastric cancer patients, and at which pathological type or stage patients will benefit most.

\section{Conclusions}

(I) In patients with gastric cancer, there is abnormal arginine metabolism, and the blood arginine level is significantly higher than that of patients with benign gastric diseases. The OS of gastric cancer patients with high arginine expression is longer, which means arginine can be used as a marker to evaluate the prognosis of gastric cancer.

(II) Elevated levels of metabolism key enzyme ASS1 may be the mechanism of high serum arginine levels in gastric cancer patients, and the OS and DFS of gastric cancer patients in the group with high expression of ASS1 were longer than those in the group with low expression.

\section{Acknowledgments}

Funding: This work was supported by a grant from Foundation of Liaoning Educational Committee (No. XLYC1902026).

\section{Footnote}

Reporting Checklist: The authors have completed the REMARK reporting checklist. Available at http://dx.doi. org/10.21037/tcr-21-794

Data Sharing Statement: Available at http://dx.doi. org/10.21037/tcr-21-794

Conflicts of Interest: All authors have completed the ICMJE uniform disclosure form (available at http://dx.doi. org/10.21037/tcr-21-794). The authors have no conflicts of interest to declare.

Ethical Statement: The authors are accountable for all aspects of the work in ensuring that questions related to the accuracy or integrity of any part of the work are appropriately investigated and resolved. All procedures performed in this study involving human participants were in accordance with the Declaration of Helsinki (as revised in 2013). This study was approved by the institutional ethics committee of The First Affiliated Hospital of Jinzhou Medical University (No. 202058). All enrolled patients provided written informed consent for their specimens to be used for research purpose.

Open Access Statement: This is an Open Access article distributed in accordance with the Creative Commons Attribution-NonCommercial-NoDerivs 4.0 International License (CC BY-NC-ND 4.0), which permits the noncommercial replication and distribution of the article with the strict proviso that no changes or edits are made and the original work is properly cited (including links to both the formal publication through the relevant DOI and the license). See: https://creativecommons.org/licenses/by-nc-nd/4.0/.

\section{References}

1. Cai Z, Liu Q. Understanding the Global Cancer Statistics 2018: implications for cancer control. Sci China Life Sci 2019;62:1-4.

2. Ferlay J, Soerjomataram I, Dikshit R, et al. Cancer incidence and mortality worldwide: sources, methods and major patterns in GLOBOCAN 2012. Int J Cancer 2015;136:E359-E386.

3. DeBerardinis RJ, Thompson CB. Cellular metabolism and disease: what do metabolic outliers teach us. Cell 2012;148:1132-44.

4. Jing F, Hu X, Cao Y, et al. Discriminating gastric cancer and gastric ulcer using human plasma amino acid metabolic profile. IUBMB Life 2018;70:553-62.

5. Lario S, Ramírez-Lázaro MJ, Sanjuan-Herráez D, et al. Plasma sample based analysis of gastric cancer progression using targeted metabolomics. Sci Rep 2017;7:17774.

6. Tsai CK, Yeh TS, Wu RC, et al. Metabolomic alterations and chromosomal instability status in gastric cancer. World J Gastroenterol 2018;24:3760-9.

7. Liu HN, Wu H, Tseng YJ, et al. Serum microRNA signatures and metabolomics have high diagnostic value in gastric cancer. BMC Cancer 2018;18:415-25.

8. Corona G, Cannizzaro R, Miolo G, et al. Use of Metabolomics as a Complementary Omic Approach to Implement Risk Criteria for First-Degree Relatives of Gastric Cancer Patients, Int J Mol Sci 2018,19:750-62.

9. Morris SM. Arginine metabolism: boundaries of our 
knowledge. J Nutr 2007;137:1602S-1609S.

10. Selvi I, Basar H, Baydilli N, et al. The importance of plasma arginine level and its downstream metabolites in diagnosing prostate cancer. Int Urol Nephrol 2019;51:1975-83.

11. Hu L, Gao Y, Cao Y, et al. Association of plasma arginine with breast cancer molecular subtypes in women of Liaoning province. IUBMB Life 2016;68:980-4.

12. Zhang ZX, Qi F, Zhou DJ, et al. Effect of 5-fluorouracil in combination with Astragalus membranaceus on amino acid metabolism in mice model of gastric carcinoma. Zhonghua Wei Chang Wai Ke Za Zhi 2006;9:445-7.

13. Bian Y, Li W, Kremer DM, et al. Cancer SLC43A2 alters T cell methionine metabolism and histone methylation. Nature 2020;585:277-82.

14. Sreekumar A, Poisson LM, Rajendiran TM, et al. Metabolomic profiles delineate potential role for sarcosine in prostate cancer progression. Nature 2009;457:910-4.

15. Fiehn O. Metabolomics--the link between genotypes and phenotypes. Plant Mol Biol 2002,48:155-71.

16. Jung J, Jung Y, Bang EJ, et al. Noninvasive diagnosis and evaluation of curative surgery for gastric cancer by using NMR-based metabolomic profiling. Ann Surg Oncol 2014;21 Suppl 4:S736-742.

17. Pirzadeh M, Khalili N, Rezaei N. The interplay between aryl hydrocarbon receptor, H. pylori, tryptophan, and arginine in the pathogenesis of gastric cancer. Int Rev Immunol 2020. [Epub ahead of print]. doi: 10.1080/08830185.2020.1851371.

18. Wang D, Li W, Zou Q, et al. Serum metabolomic profiling of human gastric cancer and its relationship with the prognosis. Oncotarget 2017;8:110000-15.

19. Feldmeyer N, Wabnitz G, Leicht S, et al. Arginine deficiency leads to impaired cofilin dephosphorylation in activated human $T$ lymphocytes. Int Immunol 2012;24:303-13.

20. Yachimovich-Cohen N, Even-Ram S, Shufaro Y, et al. Human embryonic stem cells suppress $T$ cell responses via arginase I-dependent mechanism. J Immunol 2010;184:1300-8.

21. Rodriguez PC, Zea AH, DeSalvo J, et al. L-arginine consumption by macrophages modulates the expression of CD3 zeta chain in T lymphocytes. J Immunol 2003;171:1232-9.

22. Szefel J, Danielak A, Kruszewski WJ. Metabolic pathways of L-arginine and therapeutic consequences in tumors. Adv Med Sci 2019;64:104-10.

23. Pirzadeh M, Khalili N, Rezaei N. The interplay between aryl hydrocarbon receptor, H. pylori, tryptophan, and arginine in the pathogenesis of gastric cancer. Int Rev Immunol 2020:1-42.

24. Chaturvedi R, de Sablet T, Coburn LA, et al. Arginine and polyamines in Helicobacter pylori-induced immune dysregulation and gastric carcinogenesis. Amino Acids 2012;42:627-40.

25. Wu H, Xue R, Tang Z, et al. Metabolomic investigation of gastric cancer tissue using gas chromatography/mass spectrometry. Anal Bioanal Chem 2010;396:1385-95.

26. Xiao S, Zhou L. Gastric cancer: Metabolic and metabolomics perspectives (Review). Int J Oncol 2017;51:5-17.

27. Shu XL, Xu H, Yu TT, et al. Regulation of apoptosis in human gastric cancer cell line SGC-7901 by L-arginine. Panminerva Med 2014;56:227-31.

28. Nanthakumaran S, Brown I, Heys SD, et al. Inhibition of gastric cancer cell growth by arginine: molecular mechanisms of action. Clin Nutr 2009;28:65-70.

29. Ji JX, Cochrane DR, Tessier-Cloutier B, et al. Arginine Depletion Therapy with ADI-PEG20 Limits Tumor Growth in Argininosuccinate Synthase-Deficient Ovarian Cancer, Including Small-Cell Carcinoma of the Ovary, Hypercalcemic Type. Clin Cancer Res 2020;26:4402-13.

30. Delage B, Fennell DA, Nicholson L, et al. Arginine deprivation and argininosuccinate synthetase expression in the treatment of cancer. Int J Cancer 2010;126:2762-72.

31. Tao X, Zuo Q, Ruan H, et al. Argininosuccinate synthase 1 suppresses cancer cell invasion by inhibiting STAT3 pathway in hepatocellular carcinoma. Acta Biochim Biophys Sin 2019;51:263-76.

32. Hou JC, Xu Z, Zhong SL, et al. Circular RNA circASS1 is downregulated in breast cancer cells MDA-MB-231 and suppressed invasion and migration. Epigenomics 2019;11:199-213.

33. Bateman LA, Ku WM, Heslin MJ, et al. Argininosuccinate Synthase 1 is a Metabolic Regulator of Colorectal Cancer Pathogenicity. ACS Chem Biol 2017;12:905-11.

34. Nicholson LJ, Smith PR, Hiller L, et al. Epigenetic silencing of argininosuccinate synthetase confers resistance to platinum-induced cell death but collateral sensitivity to arginine auxotrophy in ovarian cancer. Int $\mathrm{J}$ Cancer 2009;125:1454-63.

35. Szlosarek PW, Grimshaw MJ, Wilbanks GD, et al. Aberrant regulation of argininosuccinate synthetase by TNF-alpha in human epithelial ovarian cancer. Int J Cancer 2007;121:6-11.

36. Ascierto PA, Scala S, Castello G, et al. Pegylated arginine 
deiminase treatment of patients with metastatic melanoma: results from phase I and II studies. J Clin Oncol 2005;23:7660-8.

37. Izzo F, Marra P, Beneduce G, et al. Pegylated arginine deiminase treatment of patients with unresectable hepatocellular carcinoma: results from phase I/II studies. J Clin Oncol 2004;22:1815-22.

38. Tsai CY, Chi HC, Chi LM, et al. Argininosuccinate synthetase 1 contributes to gastric cancer invasion and progression by modulating autophagy. FASEB J 2018;32:2601-14.

Cite this article as: Shi LY, Wang YY, Jing Y, Xu MH, Zhu ZT, Wang QJ. Abnormal arginine metabolism is associated with prognosis in patients of gastric cancer. Transl Cancer Res 2021;10(5):2451-2469. doi: 10.21037/tcr-21-794
39. Adiamah A, Skořepa P, Weimann A, et al. The Impact of Preoperative Immune Modulating Nutrition on Outcomes in Patients Undergoing Surgery for Gastrointestinal Cancer: A Systematic Review and Meta-analysis. Ann Surg 2019;270:247-56.

40. Klek S, Scislo L, Walewska E, Choruz R, Galas A. Enriched enteral nutrition may improve short-term survival in stage IV gastric cancer patients: A randomized, controlled trial. Nutrition 2017;36:46-53.

(English Language Editor: K. Brown) 